MATHEMATICS OF COMPUTATION

Volume 73, Number 246, Pages 1023-1030

S 0025-5718(03)01501-1

Article electronically published on December 19, 2003

\title{
PRIME SIEVES USING BINARY QUADRATIC FORMS
}

\author{
A. O. L. ATKIN AND D. J. BERNSTEIN
}

\begin{abstract}
We introduce an algorithm that computes the prime numbers up to $N$ using $O(N / \log \log N)$ additions and $N^{1 / 2+o(1)}$ bits of memory. The algorithm enumerates representations of integers by certain binary quadratic forms. We present implementation results for this algorithm and one of the best previous algorithms.
\end{abstract}

\section{INTRODUCTION}

Pritchard in 14 asked whether it is possible to print the prime numbers up to $N$, in order, using $o(N)$ operations and $O\left(N^{\alpha}\right)$ bits of memory for some $\alpha<1$. Here "memory" does not include the paper used by the printer. "Operations" refers to loads, stores, comparisons, additions, and subtractions of $O(\log N)$-bit integers.

The answer is yes. We present a new algorithm that uses $o(N)$ operations and $N^{1 / 2+o(1)}$ bits of memory. We also present some implementation results; the new method is useful in practice.

This paper is not the end of the story. Galway in 8 and [9] started from the method described here and replaced certain subroutines, namely the algorithms in Section 4 of this paper, with computational versions of Sierpinski's theorem on the circle problem. The resulting algorithm uses somewhat more operations but only $N^{1 / 3+o(1)}$ bits of memory.

Strategy. The idea of the sieve of Eratosthenes is to enumerate values of the reducible binary quadratic form $x y$. The idea of the new algorithm is to enumerate values of certain irreducible binary quadratic forms. For example, a squarefree positive integer $p \in 1+4 \mathbf{Z}$ is prime if and only if the equation $4 x^{2}+y^{2}=p$ has an odd number of positive solutions $(x, y)$. There are only $O(N)$ pairs $(x, y)$ such that $4 x^{2}+y^{2} \leq N$

We cover all primes $p>3$ as follows. For $p \in 1+4 \mathbf{Z}$ we use $4 x^{2}+y^{2}$ with $x>0$ and $y>0$; for $p \in 7+12 \mathbf{Z}$ we use $3 x^{2}+y^{2}$ with $x>0$ and $y>0$; for $p \in 11+12 \mathbf{Z}$ we use $3 x^{2}-y^{2}$ with $x>y>0$. Section 6 reviews the relevant facts about these quadratic forms.

(One can vary the forms and the $(x, y)$-conditions. For example, for $p \in 1+4 \mathbf{Z}$ one could use $x^{2}+y^{2}$ with $x>y>0$; for $p \in 3+8 \mathbf{Z}$ one could use $2 x^{2}+y^{2}$ with $x>0$ and $y>0$; for $p \in 7+8 \mathbf{Z}$ one could use $2 x^{2}-y^{2}$ with $x>y>0$. There are many possible choices; we have not determined the optimal set of forms.)

Received by the editor September 7, 1999 and, in revised form, March 30, 2002.

2000 Mathematics Subject Classification. Primary 11Y11; Secondary 11E25.

The second author was supported by the National Science Foundation under grant DMS9600083.

(C) 2003 A. O. L. Atkin and D. J. Bernstein 
A standard improvement in the sieve of Eratosthenes is to enumerate values of $x y$ not divisible by 2,3 , or 5 ; see Section 2 for details. This reduces the number of pairs $(x, y)$ by a constant factor. Similarly, we enumerate values of our quadratic forms not divisible by 5 ; see Section 3 for details.

More generally, one can select an integer $W$ and enumerate values relatively prime to $W$. One can save a factor of $\log \log N$ in the running time of the sieve of Eratosthenes by letting $W$ grow slowly with $N$. The same is true of the new method. In Section 5 we show that one can enumerate the primes up to $N$ using $O(N / \log \log N)$ operations and $N^{1 / 2+o(1)}$ bits of memory.

\section{The Sieve of Eratosthenes}

The following algorithm is standard. It uses $B$ bits of memory to compute the primes in an arithmetic progression of $B$ numbers.

Algorithm 2,1. Given $\delta \in\{1,7,11,13,17,19,23,29\}$, to print all primes of the form $30 k+\delta$ with $L \leq k<L+B$ :

1. Set $a_{L} \leftarrow 1, a_{L+1} \leftarrow 1, \ldots, a_{L+B-1} \leftarrow 1$.

2. For each prime $q \geq 7$ with $q^{2}<30 L+30 B$ :

3. $\quad$ For each $k$ with $30 k+\delta$ a nontrivial multiple of $q$ :

4. $\quad$ Set $a_{k} \leftarrow 0$.

5. Print $30 k+\delta$ for each $k$ with $a_{k}=1$.

"Nontrivial multiple of $q$ " in step 3 means " $m q$ for some $m>1$ " but can safely be replaced by " $m q$ for some $m \geq q$."

One can run Algorithm 2,1 for each $\delta$, and merge the results, to find all the primes $p$ with $30 L \leq p<30 L+30 B$. This uses $8 B$ bits of memory, not counting the space needed to store the set of primes $q$.

To enumerate the primes $p$ in a larger interval, say $30 L \leq p<30 L+60 B$, one can enumerate first the primes between $30 L$ and $30 L+30 B$, then the primes between $30 L+30 B$ and $30 L+60 B$, reusing the same $8 B$ bits of memory.

The number of iterations of step 4 of Algorithm 21 is approximately $B / 7$ for $q=7, B / 11$ for $q=11$, and so on. By Mertens's theorem, the sum $B \sum_{q}(1 / q)$ is roughly $B(\log \log (30 L+30 B)-1.465)$. See [10, Theorem 427].

Implementation results. The second author's implementation of Algorithm 2 1 , using the gcc 2.8 .1 compiler on an UltraSPARC-I/167, takes about $3.3 \cdot 10^{9}$ cycles to find the 50847534 primes up to $10^{9}$. Here $B=128128$; the UltraSPARC has 131072 bits of fast memory.

Notes. Singleton in [15] suggested chopping a large interval into small pieces and applying the sieve of Eratosthenes to each piece. The same idea was published independently in [4] and later in [2].

Sieving an arithmetic progression is the $p$-adic analogue of sieving a bounded interval. Presumably Eratosthenes did not bother writing down even numbers in his sieve.

Instead of running Algorithm 2.1 independently for each $\delta$, one can handle all $\delta$ simultaneously for each $q$ : find all nontrivial multiples of $q$ between $30 L$ and $30 L+30 B$, and translate each multiple into a pair $(k, \delta)$. See [12] for details. For sufficiently large $q$ this saves time despite the added cost of translation. 
One can include composite integers $q$ in step 2 of Algorithm 21. For example, it is easy to run through all integers $q>1$ with $q \bmod 30 \in\{1,7,11,13,17,19,23,29\}$. This saves the space necessary to store the primes $q$, at a small cost in time.

\section{Prime Sieves using irReducible Binary QUAdRatiC Forms}

The following algorithms are new. Each algorithm uses $B$ bits of memory to compute primes in an arithmetic progression of $B$ numbers. Algorithm 31 requires each number to be congruent to 1 modulo 4; Algorithm 32 requires each number to be congruent to 1 modulo 6; Algorithm 3.3 requires each number to be congruent to 11 modulo 12 .

Algorithm 3.1. Given $\delta \in\{1,13,17,29,37,41,49,53\}$, to print all primes of the form $60 k+\delta$ with $L \leq k<L+B$ :

1. Set $a_{L} \leftarrow 0, a_{L+1} \leftarrow 0, \ldots, a_{L+B-1} \leftarrow 0$.

2. For each $(x, y, k)$ with $x>0, y>0, L \leq k<L+B$, and $4 x^{2}+y^{2}=60 k+\delta$ :

3. $\quad$ Set $a_{k} \leftarrow 1-a_{k}$.

4. For each prime $q \geq 7$ with $q^{2}<60 L+60 B$ :

5. $\quad$ For each $k$ with $60 k+\delta$ divisible by $q^{2}$ :

6. $\quad$ Set $a_{k} \leftarrow 0$.

7. Print $60 k+\delta$ for each $k$ with $a_{k}=1$.

Steps 2 and 3 count, for each $k$, the parity of the number of pairs $(x, y)$ with $4 x^{2}+y^{2}=60 k+\delta$. Theorem 6.1 says that $60 k+\delta$ is prime if and only if the number of pairs is odd and $60 k+\delta$ is squarefree. Steps 4,5 , and 6 eliminate each $k$ for which $60 k+\delta$ is not squarefree.

The condition $4 x^{2}+y^{2} \in \delta+60 \mathbf{Z}$ in step 2 implies 16 possibilities (depending on $\delta)$ for $(x \bmod 15, y \bmod 30)$. Each possibility can be handled by Algorithm 41 below. There are approximately $(4 \pi / 15) B$ iterations of step 3 .

Algorithm 3,2. Given $\delta \in\{1,7,13,19,31,37,43,49\}$, to print all primes of the form $60 k+\delta$ with $L \leq k<L+B$ :

1. Set $a_{L} \leftarrow 0, a_{L+1} \leftarrow 0, \ldots, a_{L+B-1} \leftarrow 0$.

2. For each $(x, y, k)$ with $x>0, y>0, L \leq k<L+B$, and $3 x^{2}+y^{2}=60 k+\delta$ :

3. Set $a_{k} \leftarrow 1-a_{k}$.

4. For each prime $q \geq 7$ with $q^{2}<60 L+60 B$ :

5. For each $k$ with $60 k+\delta$ divisible by $q^{2}$ :

6. $\quad$ Set $a_{k} \leftarrow 0$.

7. Print $60 k+\delta$ for each $k$ with $a_{k}=1$.

Algorithm [3.2 is justified by Theorem 6.2. In step 2 there are 12 possibilities for $(x \bmod 10, y \bmod 30)$, each of which can be handled by Algorithm 42 below. There are approximately $(\pi \sqrt{0.12}) B$ iterations of step 3 .

Algorithm 3.3. Given $\delta \in\{11,23,47,59\}$, to print all primes of the form $60 k+\delta$ with $L \leq k<L+B$ :

1. Set $a_{L} \leftarrow 0, a_{L+1} \leftarrow 0, \ldots, a_{L+B-1} \leftarrow 0$.

2. For each $(x, y, k)$ with $x>y>0, L \leq k<L+B$, and $3 x^{2}-y^{2}=60 k+\delta$ :

3. Set $a_{k} \leftarrow 1-a_{k}$.

4. For each prime $q \geq 7$ with $q^{2}<60 L+60 B$ :

5. For each $k$ with $60 k+\delta$ divisible by $q^{2}$ :

6. $\quad$ Set $a_{k} \leftarrow 0$.

7. Print $60 k+\delta$ for each $k$ with $a_{k}=1$. 
Algorithm [3. 3 is justified by Theorem 6.3. In step 2 there are 24 possibilities for $(x \bmod 10, y \bmod 30)$, each of which can be handled by Algorithm 43 below. There are approximately $(\sqrt{1.92} \log (\sqrt{0.5}+\sqrt{1.5})) B$ iterations of step 3 .

Implementation results. The second author's implementation of Algorithm 31 , Algorithm 3,2, and Algorithm 3,3, using gcc 2.8.1 on an UltraSPARC-I/167 with $B=128128$, takes about $2.5 \cdot 10^{9}$ cycles to find the primes up to $10^{9}$. For the code see http://cr.yp.to/primegen.html.

About $87 \%$ of the time was spent in steps 2 and 3 of these algorithms: $38 \%$ in Algorithm 31 for $\delta \in\{1,13,17,29,37,41,49,53\} ; 26 \%$ in Algorithm 32 for $\delta \in\{7,19,31,43\} ; 23 \%$ in Algorithm 33 for $\delta \in\{11,23,47,59\}$. About half of the remaining time was spent in steps 4,5 , and 6 .

Notes. One could change the "even, odd" counter $a_{k}$ in Algorithm 31 to a "zero, one, more" counter, and then skip some values of $q$ in step 4 . The same comment applies to Algorithm 3,2 and Algorithm 3.3.

\section{EnUmerating LATtice Points}

The idea of Algorithm 4 1 is to scan upwards from the lower boundary of the first quadrant of the annulus $60 L \leq 4 x^{2}+y^{2}<60 L+60 B$. The total number of points considered by Algorithm 41 is $(1 / 450)(\pi / 8)(60 B)+O(\sqrt{60 L+60 B})$. Here $(\pi / 8)(60 B)$ is the area of the quadrant, and $1 / 450$ accounts for the restriction on $(x \bmod 15, y \bmod 30)$. Similar comments apply to Algorithm 4.2 and Algorithm 443.

Algorithm 4,1. Given positive integers $\delta<60, f \leq 15$, and $g \leq 30$ such that $\delta \equiv 4 f^{2}+g^{2}(\bmod 60)$, to print all triples $(x, y, k)$ with $x>0, y>0, L \leq k<L+B$, $4 x^{2}+y^{2}=60 k+\delta, x \in f+15 \mathbf{Z}$, and $y \in g+30 \mathbf{Z}:$

1. Set $x \leftarrow f, y_{0} \leftarrow g$, and $k_{0} \leftarrow\left(4 f^{2}+g^{2}-\delta\right) / 60$. (Starting in step 3 we will move $\left(x, y_{0}\right)$ along the lower boundary, from right to left, keeping track of $k_{0}=\left(4 x^{2}+y_{0}^{2}-\delta\right) / 60$. $)$

2. If $k_{0}<L+B$ : Set $k_{0} \leftarrow k_{0}+2 x+15$. Set $x \leftarrow x+15$. Repeat this step.

3. (Move left.) Set $x \leftarrow x-15$. Set $k_{0} \leftarrow k_{0}-2 x-15$. Stop if $x \leq 0$.

4. (Move up if necessary.) If $k_{0}<L$ : Set $k_{0} \leftarrow k_{0}+y_{0}+15$. Set $y_{0} \leftarrow y_{0}+30$. Repeat this step.

5. (Now $4 x^{2}+y_{0}^{2} \geq 60 L$; and if $y_{0}>30$ then $4 x^{2}+\left(y_{0}-30\right)^{2}<60 L$.) Set $k \leftarrow k_{0}$ and $y \leftarrow y_{0}$.

6. (Now $4 x^{2}+y^{2}=60 k+\delta \geq 60 L$.) If $k<L+B$ : Print $(x, y, k)$. Set $k \leftarrow k+y+15$. Set $y \leftarrow y+30$. Repeat this step.

7. Go back to step 3.

Algorithm 4,2. Given positive integers $\delta<60, f \leq 10$, and $g \leq 30$ such that $\delta \equiv 3 f^{2}+g^{2}(\bmod 60)$, to print all triples $(x, y, k)$ with $x>0, y>0, L \leq k<L+B$, $3 x^{2}+y^{2}=60 k+\delta, x \in f+10 \mathbf{Z}$, and $y \in g+30 \mathbf{Z}$ :

1. Set $x \leftarrow f, y_{0} \leftarrow g$, and $k_{0} \leftarrow\left(3 f^{2}+g^{2}-\delta\right) / 60$.

2. If $k_{0}<L+B$ : Set $k_{0} \leftarrow k_{0}+x+5$. Set $x \leftarrow x+10$. Repeat this step.

3. Set $x \leftarrow x-10$. Set $k_{0} \leftarrow k_{0}-x-5$. Stop if $x \leq 0$.

4. If $k_{0}<L$ : Set $k_{0} \leftarrow k_{0}+y_{0}+15$. Set $y_{0} \leftarrow y_{0}+30$. Repeat this step.

5. Set $k \leftarrow k_{0}$ and $y \leftarrow y_{0}$. 
6. If $k<L+B$ : Print $(x, y, k)$. Set $k \leftarrow k+y+15$. Set $y \leftarrow y+30$. Repeat this step.

7. Go back to step 3 .

Algorithm 4,3. Given positive integers $\delta<60, f \leq 10$, and $g \leq 30$ such that $\delta \equiv 3 f^{2}-g^{2}(\bmod 60)$, to print all triples $(x, y, k)$ with $x>y>0, L \leq k<L+B$, $3 x^{2}-y^{2}=60 k+\delta, x \in f+10 \mathbf{Z}$, and $y \in g+30 \mathbf{Z}$ :

1. Set $x \leftarrow f, y_{0} \leftarrow g$, and $k_{0} \leftarrow\left(3 f^{2}-g^{2}-d\right) / 60$.

2. If $k_{0} \geq L+B$ : Stop if $x \leq y_{0}$. Set $k_{0} \leftarrow k_{0}-y_{0}-15$. Set $y_{0} \leftarrow y_{0}+30$. Repeat this step.

3. Set $k \leftarrow k_{0}$ and $y \leftarrow y_{0}$.

4. If $k \geq L$ and $y<x$ : Print $(x, y, k)$. Set $k \leftarrow k-y-15$. Set $y \leftarrow y+30$. Repeat this step.

5. Set $k_{0} \leftarrow k_{0}+x+5$. Set $x \leftarrow x+10$. Go back to step 2 .

Notes. Tracing a level curve is a standard technique in computer graphics; see, e.g., [1, Chapter 17]. It is often credited to [5], but it appeared earlier in [11, Section $3]$.

\section{Asymptotic Performance}

For large $N$ one can compute the primes up to $N$ as follows.

Define $W$ as 12 times the product of all the primes from 5 up to about $\sqrt{\log N}$. Note that $W$ is in $N^{o(1)}$; it is roughly $\exp \sqrt{\log N}$.

Write $\varphi_{1}$ for the number of units modulo $W$. Then $\varphi_{1} / W$ is in $O(1 / \log \log N)$ by Mertens's theorem.

Write $\varphi_{2}$ for the number of pairs $(x \bmod W, y \bmod W)$ such that $4 x^{2}+y^{2}$ or $3 x^{2}+y^{2}$ or $3 x^{2}-y^{2}$ is a unit modulo $W$. Then $\varphi_{2} / W^{2}$ is in $O(1 / \log \log N)$ by a standard generalization of Mertens's theorem.

Select an integer $B$ close to $W \sqrt{N}$. The method described in this section uses $\varphi_{1} B$ bits of memory, i.e., $N^{1 / 2+o(1)}$ bits. We leave it to the reader to investigate how much the memory consumption can be reduced without noticeably affecting the number of operations.

Here is how to compute the primes in $\delta+W \mathbf{Z}$ between $W L$ and $W L+W B$, given a positive integer $L<N / W$ and given a unit $\delta$ modulo $W$ :

- Define $(a, b)=(4,1)$ if $\delta \in 1+4 \mathbf{Z}$; define $(a, b)=(3,1)$ if $\delta \in 7+12 \mathbf{Z}$; define $(a, b)=(3,-1)$ if $\delta \in 11+12 \mathbf{Z}$.

- Find all possible $(x \bmod W, y \bmod W)$ given that $a x^{2}+b y^{2} \in \delta+W \mathbf{Z}$. This can easily be done in $W^{O(1)}$ operations.

- For each possible $(x \bmod W, y \bmod W)$, enumerate all $(x, y)$ with $W L \leq$ $a x^{2}+b y^{2}<W L+W B$, as in Section 4, and toggle the appropriate bits in a $B$-bit array. The number of operations here is $O(B / W)$ for each possible $(x \bmod W, y \bmod W)$ : the choice of $B$ guarantees that $\sqrt{W L+W B}$ is in $O(B / W)$.

- Eliminate numbers that are not squarefree, as in Section 3, to determine the primes. The number of operations here is $O(B)$.

The total number of operations over all $\delta$, to compute all the primes between $W L$ and $W L+W B$ using $\varphi_{1}$ separate $B$-bit arrays, is $W^{O(1)}+O\left(\varphi_{2} B / W\right)+O\left(\varphi_{1} B\right)=$ $O(W B / \log \log N)$. 
Consequently one can compute all the primes up to $N$ using $O(N / \log \log N)$ operations and $N^{1 / 2+o(1)}$ bits of memory.

Notes. Pritchard in 14 pointed out that, by the method of Section 2, one can compute the primes up to $N$ using $O(N)$ operations and $O\left(N^{1 / 2}(\log \log N) / \log N\right)$ bits of memory.

By a similar method one can compute the primes up to $N$ using $O(N / \log \log N)$ operations and $N^{1+o(1)}$ bits of memory. Pritchard gave a proof in [12] and a simpler proof in [13]. Dunten, Jones, and Sorenson in [6] reduced the amount of memory by a logarithmic factor; of course, $N^{1+o(1)} / \log N$ is still $N^{1+o(1)}$.

The new method uses $O(N / \log \log N)$ operations with only $N^{1 / 2+o(1)}$ bits of memory. No previous method achieves both bounds simultaneously.

\section{QUADRATIC FORMS}

Theorem 6.1. Let $n$ be a squarefree positive integer with $n \in 1+4 \mathbf{Z}$. Then $n$ is prime if and only if $\#\left\{(x, y): x>0, y>0,4 x^{2}+y^{2}=n\right\}$ is odd.

The following proof uses the fact that the unit group $\mathbf{Z}[i]^{*}$ of the principal ideal domain $\mathbf{Z}[i]$, where $i=\sqrt{-1}$, is $\{1,-1, i,-i\}$. The idea is to find representatives in $\mathbf{Z}[i]$ for the semigroup $\mathbf{Z}[i] / \mathbf{Z}[i]^{*}$.

Proof. The statement is true for $n=1$, so assume $n>1$.

Define $S=\left\{(x, y): y>0,4 x^{2}+y^{2}=n\right\}$. Define $T$ as the set of norm- $n$ ideals in $\mathbf{Z}[i]$. For each $(x, y) \in S$ define $f(x, y) \in T$ as the ideal generated by $y+2 x i$.

Step 1: $f$ is injective. Indeed, the other generators of the ideal generated by $y+2 x i$ are $-y-2 x i,-2 x+y i$, and $2 x-y i$, none of which are of the form $y^{\prime}+2 x^{\prime} i$ with $y^{\prime}>0$.

Step 2: $f$ is surjective. Indeed, take any $I \in T$. Select a generator $a+b i$ of $I$; then $a^{2}+b^{2}=n$. Note that $b \neq 0$ since $n$ is squarefree. If $a$ is even and $b>0$ then $I=f(-a / 2, b)$; if $a$ is even and $b<0$ then $I=f(a / 2,-b)$; if $a$ is odd and $a>0$ then $I=f(b / 2, a)$; if $a$ is odd and $a<0$ then $I=f(-b / 2,-a)$.

Step 3: If $n$ is prime then $\# T=2$ so $\#\left\{(x, y): x>0, y>0,4 x^{2}+y^{2}=n\right\}=$ $(\# S) / 2=(\# T) / 2=1$. Otherwise write $n=p_{1} p_{2} \cdots p_{r}$ where each $p_{k}$ is prime. The number of norm- $p_{k}$ ideals is even, so \#T is divisible by $2^{r}$, hence by 4 ; thus $\#\left\{(x, y): x>0, y>0,4 x^{2}+y^{2}=n\right\}=(\# S) / 2=(\# T) / 2$ is even.

Theorem 6.2. Let $n$ be a squarefree positive integer with $n \in 1+6 \mathbf{Z}$. Then $n$ is

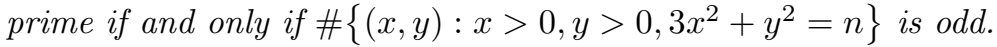

The following proof uses the fact that the unit group of the principal ideal domain $\mathbf{Z}[\omega]$, where $\omega=(-1+\sqrt{-3}) / 2$, is $\left\{1, \omega, \omega^{2},-1,-\omega,-\omega^{2}\right\}$.

Proof. Assume $n>1$. Define $S=\left\{(x, y): y>0,3 x^{2}+y^{2}=n\right\}$. Define $T$ as the set of norm- $n$ ideals in $\mathbf{Z}[\omega]$. For each $(x, y) \in S$ define $f(x, y) \in T$ as the ideal generated by $x+y+2 x \omega$. If $n$ is prime then $\# T=2$; otherwise $\# T$ is divisible by 4. By calculations similar to those in Theorem 6.1 the reader may verify that $f$ is a bijection from $S$ to $T$.

Theorem 6.3. Let $n$ be a squarefree positive integer with $n \in 11+12 \mathbf{Z}$. Then $n$ is prime if and only if $\#\left\{(x, y): x>y>0,3 x^{2}-y^{2}=n\right\}$ is odd. 
The following proof uses the fact that the unit group $\mathbf{Z}[\gamma]^{*}$ of the principal ideal domain $\mathbf{Z}[\gamma]$, where $\gamma=\sqrt{3}$, is $\left\{ \pm(2+\gamma)^{j}: j \in \mathbf{Z}\right\}$.

Proof. Define $S=\left\{(x, y):|x|>y>0,3 x^{2}-y^{2}=n\right\}$. Define $T$ as the set of norm$n$ ideals in $\mathbf{Z}[\gamma]$. For each $(x, y) \in S$ define $f(x, y) \in T$ as the ideal generated by $y+x \gamma$. As above it suffices to show that $f$ is a bijection from $S$ to $T$.

Define $L=\log (2+\gamma)$, and define a homomorphism $\log : \mathbf{Q}[\gamma]^{*} \rightarrow \mathbf{R}^{2}$ by $\log (a+b \gamma)=(\log |a+b \gamma|, \log |a-b \gamma|)$. Then $\log \mathbf{Z}[\gamma]^{*}=(L,-L) \mathbf{Z}$. Note that if $|b|>a>0$ then $|u-v|<L$ where $(u, v)=\log (a+b \gamma)$; and if $|u-v| \leq L$ then either $|a| \leq|b|$ or $|a| \geq 3|b|$.

Injectivity: For $(x, y) \in S$ and $\left(x^{\prime}, y^{\prime}\right) \in S$ write $(u, v)=\log (y+x \gamma)$ and $\left(u^{\prime}, v^{\prime}\right)=\log \left(y^{\prime}+x^{\prime} \gamma\right)$. Then $|u-v|<L$ and $\left|u^{\prime}-v^{\prime}\right|<L$, so $\left|u-v-u^{\prime}+v^{\prime}\right|<$ $2 L$. Now assume that $f(x, y)=f\left(x^{\prime}, y^{\prime}\right)$. Then $(u, v)-\left(u^{\prime}, v^{\prime}\right) \in(L,-L) \mathbf{Z}$, so $(u, v)=\left(u^{\prime}, v^{\prime}\right)$, so $\left(x^{\prime}, y^{\prime}\right) \in\{(x, y),(-x,-y)\}$; but $y$ and $y^{\prime}$ are both positive, so $\left(x^{\prime}, y^{\prime}\right)=(x, y)$.

Surjectivity: Given a norm- $n$ ideal $I$, pick a generator $a+b \gamma$ of $I$. Write $(u, v)=$ $\log (a+b \gamma)$. Select an integer $j$ within $1 / 2$ of $(v-u) / 2 L$, and write $y+x \gamma=$ $(a+b \gamma)(2+\gamma)^{j}$. Then $\log (y+x \gamma)=(u+j L, v-j L)$, and $|(u+j L)-(v-j L)| \leq L$, so $|y| \leq|x|$ or $|y| \geq 3|x|$. But $n= \pm\left(3 x^{2}-y^{2}\right)$, and $n \in 11+12 \mathbf{Z}$, so $n=3 x^{2}-y^{2}$; in particular $3 x^{2}-y^{2}>0$ so $|y| \leq|x|$. Also $|y| \neq 0$ and $|y| \neq|x|$ since $n$ is squarefree. If $y>0$ then $I=f(x, y)$; if $y<0$ then $I=f(-x,-y)$.

Notes. These theorems are standard. See, e.g., [16, Chapter 11]. We have included proofs for the sake of completeness.

The function Log in the proof of Theorem 6.3 is an example of Dirichlet's log map. See, e.g., [7, page 169].

The approximations $(4 \pi / 15) B,(\pi \sqrt{0.12}) B$, and $(\sqrt{1.92} \log (\sqrt{0.5}+\sqrt{1.5})) B$ for the number of lattice points considered in Section 3 and Section 4 are examples of Dirichlet's analytic class-number formula. See, e.g., 7] pages 283-294], particularly 7. page 289].

\section{REFERENCES}

[1] Michael Abrash, Zen of graphics programming, Coriolis Group, Scottsdale, Arizona, 1995.

[2] Carter Bays, Richard H. Hudson, The segmented sieve of Eratosthenes and primes in arithmetic progressions to $10^{12}$, BIT 17 (1977), 121-127. MR 56:5405

[3] Wieb Bosma (editor), Algorithmic number theory: ANTS-IV, Lecture Notes in Computer Science, 1838, Springer-Verlag, Berlin, 2000. MR 2002d:11002

[4] Richard P. Brent, The first occurrence of large gaps between successive primes, Mathematics of Computation 27 (1973), 959-963. MR 48:8360. Available from http://web.comlab.ox.ac.uk/oucl/work/richard.brent/pub/pub019.html

[5] Jack Bresenham, A linear algorithm for incremental digital display of circular arcs, Communications of the ACM 20 (1977), 100-106.

[6] Brian Dunten, Julie Jones, Jonathan Sorenson, A space-efficient fast prime number sieve, Information Processing Letters 59 (1996), 79-84. MR 97g:11141

[7] Albrecht Fröhlich, Martin J. Taylor, Algebraic number theory, Cambridge University Press, Cambridge, 1991. MR 94d:11078

[8] William F. Galway, Dissecting a sieve to cut its need for space, in [3] (2000), 297-312. MR 2002g:11176

[9] William F. Galway, Analytic computation of the prime-counting function, Ph.D. thesis, University of Illinois at Urbana-Champaign, 2001.

[10] G. H. Hardy, E. M. Wright, An introduction to the theory of numbers, 5th edition, Oxford University Press, 1979. MR 81i:10002 
[11] Herbert B. Keller, J. R. Swenson, Experiments on the lattice problem of Gauss, Mathematics of Computation 17 (1963), 223-230. MR 29:3445

[12] Paul Pritchard, A sublinear additive sieve for finding prime numbers, Communications of the ACM 24 (1981), 18-23. MR 82c:10011

[13] Paul Pritchard, Explaining the wheel sieve, Acta Informatica 17 (1982), 477-485. MR 84g:10015

[14] Paul Pritchard, Fast compact prime number sieves (among others), Journal of Algorithms 4 (1983), 332-344. MR 85h:11080

[15] Richard C. Singleton, Algorithm 357: an efficient prime number generator, Communications of the ACM 12 (1969), 563-564.

[16] J. V. Uspensky, Max A. Heaslet, Elementary number theory, McGraw-Hill, New York, 1939. MR 1:38d

Department of Mathematics, Statistics, and Computer Science (M/C 249), The University of Illinois at Chicago, Chicago, Illinois 60607-7045

E-mail address: aolatkin@uic.edu

Department of Mathematics, Statistics, and Computer Science (M/C 249), The UniVersity of Illinois at Chicago, Chicago, Illinois 60607-7045

E-mail address: djb@pobox.com 